\title{
Mathematical model of brain tumour with glia-neuron interactions and chemotherapy treatment
}

\author{
Kelly C. Iarosz ${ }^{a, *}$, Fernando S. Borges ${ }^{\mathrm{b}}$, Antonio M. Batista ${ }^{\mathrm{a}, \mathrm{b}, \mathrm{c}}$, Murilo S. Baptista $^{\mathrm{a}}$, Regiane A. N. Siqueira ${ }^{\mathrm{b}}$, Ricardo \\ L. Viana ${ }^{\mathrm{d}}$, Sergio R. Lopes ${ }^{\mathrm{d}}$

\begin{abstract}
${ }^{a}$ Institute for Complex Systems and Mathematical Biology, University of Aberdeen, AB24 3UE, Aberdeen, UK, EU
${ }^{b}$ Pós-Graduação em Ciências/Física, Universidade Estadual de Ponta Grossa, 84030-900, Ponta Grossa, PR, Brazil

${ }^{d}$ Departamento de Física, Universidade Federal do Paraná, 81531-990, Curitiba, PR, Brazil
\end{abstract} \\ ${ }^{c}$ Universidade Estadual de Ponta Grossa, 84030-900, Ponta Grossa, PR, Brazil, Departamento de Matemática e Estatística
}

\begin{abstract}
In recent years, it became clear that a better understanding of the interactions among the main elements involved in the cancer network is necessary for the treatment of cancer and the suppression of cancer growth. In this work we propose a system of coupled differential equations that model brain tumour under treatment by chemotherapy, which considers interactions among the glial cells, the glioma, the neurons, and the chemotherapeutic agents. We study the conditions for the glioma growth to be eliminated, and identify values of the parameters for which the inhibition of the glioma growth is obtained with a minimal loss of healthy cells.
\end{abstract}

Keywords: brain, glioma, chemotherapy

\section{Introduction}

Cells growth is a phenomenon that has been studied in the fields of mathematics, biology, and physics (Adam \& Bellomo, 1996; Wolpert et al., 2002; Hirt et al., 2014). Unregulated cells growth may be associated with a wide group of diseases, where cells become a lump or cause illness. As a result, several growth models related to tumours have appeared in the literature (Mechón \& Condat, 2008; Aroesty et al., 1973), such as models for the metastasis (Pinho et al., 2002), the lack of nutrients (Scaleranpdi et al., 1999), the competition for resources, and the cytotoxic activity produced by the immune response (Cattani \& Ciancio, 2008; Wheldon, 1988).

The most common malignant intrinsic primary tumours of the adult human brain are the gliomas (Inaba et al., 2011). Gliomas are tumours of the neoplastic glial cells. They are classified by the World Health Organisation as oligodendroglioma, astrocytoma, mixed oligoastrocytoma, and ependymoma (Louis et al., 2007; Goodenberger \& Jenkins, 2012). Glioma causes regional effects by invasion, compression, and destruction of brain parenchyma, arterial and venous hypoxia (Cuddapah et al., 2014). There is release and recruitment of cellular mediators which disrupt normal parenchymal function (Ye \& Sontheimer, 1999). Glioma cells migrate along blood vessels, displacing the junction among glial cells and blood vessels. This way, the glioma cells can extract nutrients from the bloodstream.

\footnotetext{
* Corresponding author

Email address: kiarosz@gmail.com (Kelly C. Iarosz)
}

Displacements produce disruptions of glial functions, compromising adequate delivery of glucose and oxygen to neurons (Cuddapah et al., 2014). Moreover, disruptions of glial cells affect the neurons, because they are responsible for delivering nutrients, to provide structural support to them (Glees, 1955), and to control the biochemical compositions of the fluid surrounding the neurons. The neurons are the main responsible for the information processing from external and internal environments (Otis \& Sofronie, 2008; Fieldes, 2006; Shaham, 2005). However, glial cells are also responsible for the processing of information by mediating the neural signal. Neurons and their synapses fail to function without glial cells.

Mathematical modelling of glioma is an extensively explored area with a large variety of mathematical models exploring multiple complexities. An approach to modelling glioma is to use differential equations for the total of cells. In this case, the model ignores the spatial aspects. Kronik and collaborators (Kronik et al., 2007) proposed a mathematical model using differential equations for glioma and the immune system interactions. They incorporated studies about improved immunotherapy schedules and interventions which can lead to a cure of glioma. There are models that consider the spatio-temporal evolution, such as partial differential equations (Harpold \& Swanson, 2007) and cellular automaton (Alarcón et al., 2003) since the evolution of glioma critically depends on spatial geometry.

In this paper, we propose a mathematical model using differential equations for the growth of glioma, where the glioma cells attack the glial cells (Bulstrode et al., 2012). 
Glioma rises from glial cells (Weille, 2014), and glioma cells never return to be glial cells, resulting in invasion and destruction of surrounding healthy tissue (Alberts et al., 1994; Hahn \& Weiberg, 2002). In our model, we consider interactions among glial cells, neurons, glioma cells, and the chemotherapeutic agent. The novelty of our model was to introduce the interaction between glial cells and neurons. This interaction is biologically relevant since glial cells make crucial contributions to the formation, operation and adaptation of neural cells. Glial cells are essential for neuronal survival, once their removal causes neuronal death (Allen \& Barres, 2009). With this in mind, the main features of our model are: (i) treatment will likely preserve glial cells, (ii) glioma can be eliminated, but not without also destroying neurons. If the treatment is ceased without the complete elimination of glioma cells, concentration of glioma cells increases, (iii) there is an optimal duration for the treatment that reduces significantly the number of glioma cells by preserving the levels of glial cells and minimising the impact on the neural populations.

A major impediment to chemotherapy delivery for the glioma is the blood brain barrier (BBB). The BBB is a unique physiological structure that regulates the movement of ions, molecules, cells between the brain tissue and the blood (Gao \& Li, 2014). It is necessary to deliver antiglioma drugs across the intact BBB to obtain an efficient treatment of glioma (Srimanee et al., 2014). There are chemotherapeutic agents that are capable of penetrating the BBB (Friedman et al., 2000). Yang and collaborators (Yang et al., 2014) showed blood-brain barrier disruption through ultrasound for targeted drug delivery. Moreover, phenotypic heterogeneity of glioma contributes to failure of chemotherapy (Burrel et al., 2013). Gerlee and Nelander (Gerlee \& Nelander, 2012) studied the impact of phenotypic switching on glioma growth and invasion.

\section{Brain tumour model}

Figure 1 shows a diagram illustrating the many agents, and their interactions being considered in our model. The glioma cells only attack the glial cells. Neurons are not attacked by glioma cells, and they interact with glial cells. The chemotherapeutic agent behaves as a predator acting on all cells (Schuette, 2004).

There have been relevant studies that model the time and space evolution of gliomas. However, as mixed effect modelling techniques can not be yet applied to spatiotemporal equations (Ribba et al., 2012), then we have consider differential equations aiming to yield a simplified description of the biological process according to schematic representation which it is showed in Figure 1. Our mathematical model describes the cells concentration, and the concentration of chemotherapeutic agent. Due to mixed effects we leave out spatial considerations, and our model is a new approach to modelling the dynamic evolution of the cells concentration in a brain tumour with glia-neuron interactions.

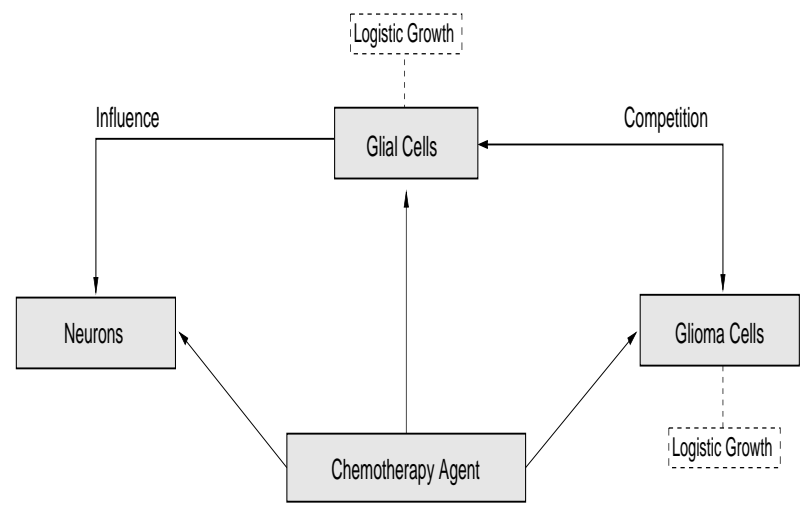

Figure 1: Schematic representation of the agents (in gray coloured boxes), and their interactions (links) considered in our model.

Our model is described by

$$
\begin{aligned}
\frac{d G(t)}{d t}= & \Omega_{1} G(t)\left(1-\frac{G(t)}{K}\right)-\Psi_{1} G(t) C(t) \\
& -\frac{P_{1} G(t) Q(t)}{A_{1}+G(t)}, \\
\frac{d C(t)}{d t}= & \Omega_{2} C(t)\left(1-\frac{C(t)}{K}\right)-\Psi_{2} G(t) C(t) \\
& -\frac{P_{2} C(t) Q(t)}{A_{2}+C(t)}, \\
\frac{d N(t)}{d t}= & \psi \dot{G}(t) H(-\dot{G}) N(t)-\frac{P_{3} N(t) Q(t)}{A_{3}+N(t)}, \\
\frac{d Q(t)}{d t}= & \Phi-\zeta Q(t),
\end{aligned}
$$

where $G$ represents the glial cells concentration (in $\mathrm{kg} / \mathrm{m}^{3}$ ), $C$ represents the glioma cells concentration (in $\mathrm{kg} / \mathrm{m}^{3}$ ), $N$ the neurons cells concentration (in $\mathrm{kg} / \mathrm{m}^{3}$ ), $Q$ is the concentration of the chemotherapeutic agent (in $\mathrm{mg} / \mathrm{m}^{2}$ ), and $H(x)$ is the Heaviside function, defined as

$$
H(x)= \begin{cases}0, & x<0 \\ \frac{1}{2}, & x=0 \\ 1, & x>0\end{cases}
$$

Table 1 shows the parameters that we consider. In Eqs. (1) and (2), the first term is the logistic growth, the second term is the interaction between glial and glioma cells. This term is due to microglia cells, that are a type of glia, which act creating on active immune defense. They have the ability to generate innate and adaptive immune responses (Yang et al., 2010). The glioma is attacked by microglia, and as a result the glioma cells discharge immune suppressive factor to defend it by paralysing the immune effector mechanism (Ghosh \& Chaudhuri, 2010). The last term of the Eqs. (1) and (2) is the effect of the chemotherapeutic agent. We consider that the chemotherapy acts killing the cells with different intensities according to the Holling type 2 killing functions. Holling (Holling, 1965) suggested kinds of functional responses to model phenomena of predation. Holling found that the predator has a Holling 2 functional 
response by taking into account the time a predator takes to handle the prey it has captured (Pei et al., 2005). The first term of Eq. (3) is related with the decrease in the neural population due to glial cells death, and the second term is the interaction with the chemotherapeutic agent. Equation (4) describes the dynamics of the chemotherapeutic agent, presenting an exponential decay in concentration. The agent rate $\zeta$ in this equation is associated with the decrease of chemotherapy that is delivered to the cells. As a result of the washout, and mainly by the blood brain barrier which prevents entry of chemotherapeutic agents into the brain.

Table 1: Description of the parameters according to literature.

\begin{tabular}{|c|c|c|c|}
\hline Description & Parameter & Values & Comment \\
\hline Proliferation & $\begin{array}{ll}\Omega_{1} & \text { for } \\
\text { GCs } & \\
\Omega_{2} & \text { for } \\
\text { CCs } & \end{array}$ & $\begin{array}{l}0.0068 \text { day }^{-1} \\
0.012 \text { day }^{-1}\end{array}$ & $\begin{array}{lll}\Omega_{1}< & \Omega_{2} \\
(\text { Pinho et } & \text { al., } \\
2013) & & \\
\text { Reference } & \\
(\text { Spratt } & \& \\
\text { Spratt, } & 1964)\end{array}$ \\
\hline $\begin{array}{ll}\text { Loss } & \text { influ- } \\
\text { ences }\end{array}$ & $\begin{array}{l}\psi \text { for } N \\
\text { due } G C s s\end{array}$ & $0-0.02$ & $\begin{array}{l}\text { Reference } \\
\text { (Pinho et al., } \\
2013)\end{array}$ \\
\hline $\begin{array}{l}\text { Interaction } \\
\text { coefficients }\end{array}$ & $\begin{array}{l}P_{1} \text { for } \\
\text { GCs } \\
P_{2} \text { for } \\
\mathrm{CCs} \\
P_{3} \text { for } \mathrm{N}\end{array}$ & $\begin{array}{l}2.4 \quad \times 10^{-5} \\
\mathrm{~m}^{2}(\mathrm{mg} . \\
\text { day })^{-1} \\
2.4 \times 10^{-2} \\
\mathrm{~m}^{2}(\mathrm{mg} . \\
\text { day })^{-1} \\
2.4 \times 10^{-5} \\
\mathrm{~m}^{2}(\mathrm{mg} . \\
\text { day })^{-1}\end{array}$ & $\begin{array}{l}\text { Reference } \\
\text { (Pinho et al., } \\
2013) \\
P_{2}>\end{array}$ \\
\hline Chemotherapy & $\begin{array}{l}\Phi \text { for in- } \\
\text { fusion } \\
\zeta\end{array}$ & $\begin{array}{l}0-150 \mathrm{mg} \\
\left(\mathrm{m}^{2} \cdot \text { day }\right)^{-1} \\
0.2 \text { day }^{-1}\end{array}$ & 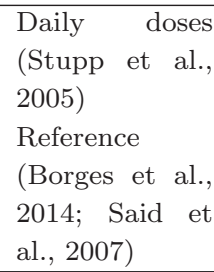 \\
\hline $\begin{array}{l}\text { Holling type } \\
2\end{array}$ & $A_{1}, A_{2}, A_{3}$ & 510 & $\begin{array}{l}A_{1}=A_{2}= \\
A_{3}=K_{i}\end{array}$ \\
\hline $\begin{array}{l}\text { Competition } \\
\text { coefficients }\end{array}$ & $\begin{array}{l}\Psi_{1} \text { be- } \\
\text { tween } \\
\text { GCs and } \\
\text { CCs } \\
\Psi_{2} \text { be- } \\
\text { tween } \\
\text { CCs and } \\
\text { GCs }\end{array}$ & $\begin{array}{l}\begin{array}{l}3.6 \times 10^{-5} \\
\text { day }^{-1}\end{array} \\
\begin{array}{l}3.6 \times 10^{-6} \\
\text { day }^{-1}\end{array}\end{array}$ & $\begin{array}{l}\text { Cancer hy- } \\
\text { pothesis } \\
\text { (Pinho et al., } \\
2013 \text { ) } \\
\Psi_{2}<\Psi_{1}\end{array}$ \\
\hline $\begin{array}{l}\text { Carrying ca- } \\
\text { pacity }\end{array}$ & $K_{1}, K_{2}, K_{3}$ & $510 \mathrm{~kg} / \mathrm{m}^{3}$ & $\begin{array}{l}\text { Reference } \\
\text { (Azevedo } \\
\text { al., 2009) }\end{array}$ \\
\hline
\end{tabular}

Glial cells are required, both in vitro and in vivo, for the survival of the neurons with which they interact. Removal of glial cells results in neuronal death, unless specific survival factors are added (Meyer-Franke et al, 1995). There are studies showing that neuron death in the ab- sence of glial is best fit by an exponential decay. Clarke and collaborators (Clarke et al., 2000) verified exponential decay in the kinetics of neuronal death in 12 models of photo-receptor degeneration, hippocampal neurons undergoing excitotoxic cell death, a mouse model of cerebellar degeneration, and Parkinson's and Huntington's diseases. Therefore, our main contribution in this work is to model the dependence of neurons on the glial cells, described by the term $\psi \dot{G}(t) H(-\dot{G}) N(t)$, in Eq. (3). This term represents an exponentially decaying function of $N(t)$, given by $N(t)=\exp (\Lambda G(t))$, where $\Lambda=\psi H($.$) . When the glial$ concentration decreases, $\dot{G}$ becomes negative, making this term to contribute negatively to $N(t)$, leading to a decrease in the neuron concentration. Whereas, the term is null if the rate of glial concentration, $\dot{G}$, is null or positive. Therefore, a decrease in the glial concentration causes death of neurons, whereas an increase does not contribute to a change in the neural population.

Recent years have seen a surge in studies about glialneuronal interaction, providing insights into the role of glial cells in neuronal function (Tiwari et al., 2014; Nakae et al., 2014). With this in mind, we consider in this work the glial-neuronal interaction due to the fact that the glioma has the origin of glial cells which are active participants in nervous system. We believe that our model is a first step towards advanced modeling techniques treating brain tumour considering glia-neuronal interactions, which in turn may have relevant impact both on tumour research and on clinical practice.

Introducing the normalised variables

$$
g=\frac{G}{K_{1}}, \quad c=\frac{C}{K_{2}}, \quad n=\frac{N}{K_{3}},
$$

where $K_{i}$ is the carrying capacity of the glial, glioma, and neural cells, respectively, we obtain the normalised mathematical model

$$
\begin{aligned}
\frac{d g(t)}{d t}= & \Omega_{1} g(t)(1-g(t))-\beta_{1} g(t) c(t) \\
& -\frac{p_{1} g(t) Q(t)}{a_{1}+g(t)}, \\
\frac{d c(t)}{d t}= & \Omega_{2} c(t)(1-c(t))-\beta_{2} g(t) c(t) \\
& -\frac{p_{2} c(t) Q(t)}{a_{2}+c(t)}, \\
\frac{d n(t)}{d t}= & \alpha \dot{g}(t) H(-\dot{g}) n(t)-\frac{p_{3} n(t) Q(t)}{a_{3}+n(t)}, \\
\frac{d Q(t)}{d t}= & \Phi-\zeta Q(t),
\end{aligned}
$$

with

$$
\begin{array}{r}
\beta_{1}=\Psi_{1} K_{2}, \quad \beta_{2}=\Psi_{2} K_{1}, \quad \alpha=\psi K_{1}, \\
a_{1}=\frac{A_{1}}{K_{1}}, \quad a_{2}=\frac{A_{2}}{K_{2}}, \quad a_{3}=\frac{A_{3}}{K_{3}} \\
p_{1}=\frac{P_{1}}{K_{1}}, \quad p_{2}=\frac{P_{2}}{K_{2}}, \quad p_{3}=\frac{P_{3}}{K_{3}},
\end{array}
$$


where Table 2 exhibits the values of the normalised parameters. The normalised model provides variables that reflect the relative density of a population of cells with respect to the mass density of the brain approximately $K=510 \mathrm{~kg} / \mathrm{m}^{3}$. For example, $n=0.3$ would mean $30 \%$ of total number of neurons that an individual could have. This normalised model allows to make comparative analysis when the model is used to simulate glioma grown in different individuals. A healthy individual, in this normalised model, would be described by the state variables $g=1, c=0$, and $n=1$.

Table 2: Values of the normalised parameters.

\begin{tabular}{c|c}
\hline Parameters & Values \\
\hline$\beta_{1}$ & $1.8 \times 10^{-2}$ day $^{-1}$ \\
$\beta_{2}$ & $1.8 \times 10^{-3}$ day $^{-1}$ \\
$\alpha$ & $0.0-10.0$ \\
$a_{1}=a_{2}=a_{3}$ & 1.0 \\
$p_{1}=p_{3}$ & $4.7 \times 10^{-8} \mathrm{~m}^{2}(\mathrm{mg} \cdot \text { day })^{-1}$ \\
$p_{2}$ & $4.7 \times 10^{-5} \mathrm{~m}^{2}(\mathrm{mg} \cdot \text { day })^{-1}$ \\
\hline
\end{tabular}

Firstly, we check the behaviour of the glioma without the infusion of a chemotherapeutic agent. Since there is no treatment the glioma cells kill the glial cells (Fig. 2a), while the glioma cells grow (Fig. 2b). Without the glial support, the neurons die (Fig. 2c). For $t=500$ there are around $34 \%$ of glial cells, and approximately $27 \%$ of neurons. Due to the logistic growth of the glial cells, they resist longer than the neurons from the attack of glioma. As a result, without chemotherapy (Fig. 2d) the glioma cells are going to kill all the cells (Figs. 2a, and c).
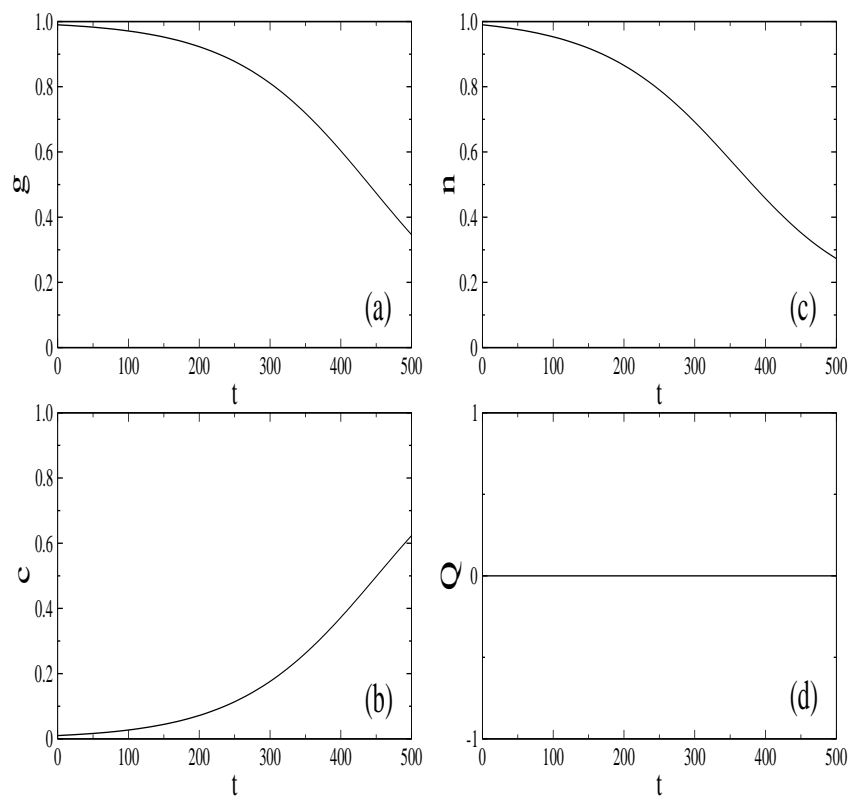

Figure 2: Temporal evolution of the concentration of (a) glial cells, (b) glioma cells, (c) neurons and (d) chemotherapeutic agent $(\Phi=0)$. We consider $g(0)=0.99, c(0)=0.01, n(0)=0.99, Q(0)=0.0$, and parameters according to Table 2 .
Then, we use the infusion of a chemotherapeutic agent in order to suppress the glioma growth. Figure 3 shows a case where our model exhibits a suppressed state. In the interval $0<t<50$ the glial cells concentration decreases (Fig. 3a) due to the attack of glioma and the presence of the chemotherapeutic agent, as well as, for $t>50$ we have $c<0.005$ (Fig. 3b). Figure 3(c) exhibits a fast decay of the neurons due to the effect of the chemotherapeutic agent on the neuron population and the decay of glial cells. Glial cells recover their normal concentration level which slows down the decay of neuron cells. If the chemotherapeutic agent is not suspended it will eventually kill all neurons, since in our model we are not considering the neurogenesis. The chemotherapeutics induce neurons death is a side effect known as neurotoxicity. Lomustine, cisplatin, topotecan, and vincristine are antitumour agents that induce cell death. Antje Wick and collaborators (Wick et al., 2004, 2009) analysed the effect of these drugs on the neurons. They verified which drugs lead to cell death in cerebellar granule neurons in a concentration dependent manner.
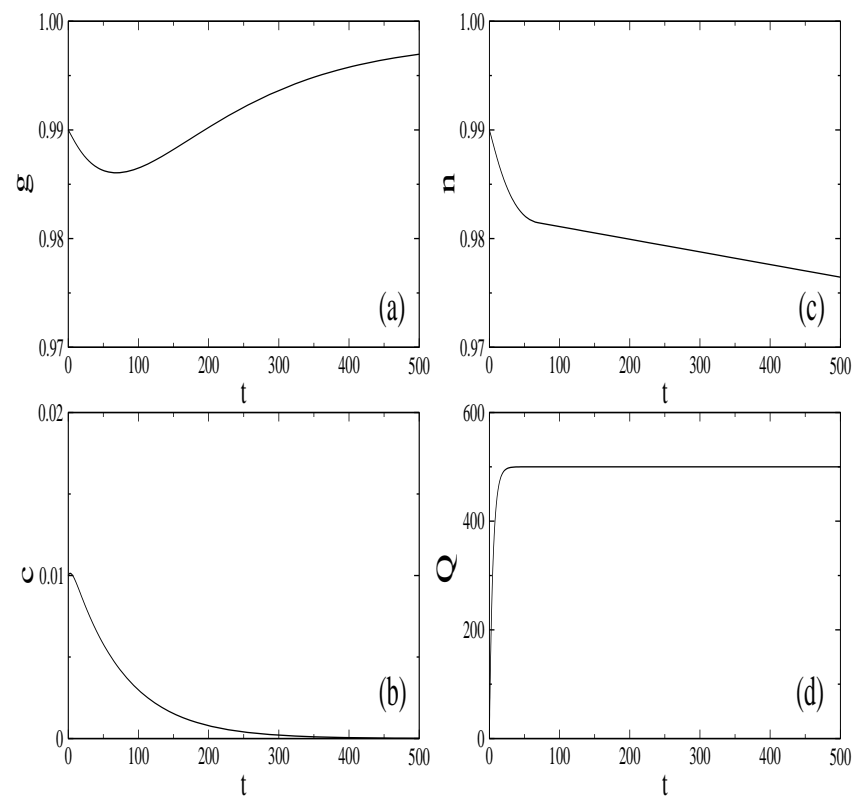

Figure 3: Temporal evolution of the concentration of (a) glial cells, (b) glioma cells, (c) neurons and (d) chemotherapy, continuous treatment $\Phi=100$. We consider $g(0)=0.99, c(0)=0.01, n(0)=0.99$, $Q(0)=0.0$ and parameters according to Table 2 .

\section{Local stability}

The study of the local stability is important to verify if the suppression of glioma is stable or unstable, or to understand whether a non desired state is stable. The model has some equilibria points $E(\bar{g}, \bar{c}, \bar{n}, \bar{Q})$. They are solution of the system $\dot{g}(t)=0, \dot{c}(t)=0, \dot{n}(t)=0, \dot{Q}(t)=0$. We consider the equilibria points physiologically feasible.

Now, we analyse the local stability for an undesirable equilibrium, where this equilibrium is $E_{0}\left(0,0,0, \Phi \zeta^{-1}\right)$. 
The eigenvalues of the Jacobian matrix are

$$
\begin{aligned}
& \lambda_{1}^{(0)}=\Omega_{1}-\frac{p_{1} \Phi}{\zeta a_{1}} \\
& \lambda_{2}^{(0)}=\Omega_{2}-\frac{p_{2} \Phi}{\zeta a_{2}} \\
& \lambda_{3}^{(0)}=-\frac{p_{3} \Phi}{\zeta a_{3}} \\
& \lambda_{4}^{(0)}=-\zeta .
\end{aligned}
$$

Through the sign of the real part of each eigenvalue we can check the stability of the equilibrium. In a hyperbolic equilibrium, if the real part of each eigenvalue is strictly negative, then the equilibrium is locally asymptotically stable. If positive, then the equilibrium is unstable. In order to ensure the stability of $E_{0}\left(0,0,0, \Phi \zeta^{-1}\right)$ it is necessary that

$$
\Phi>\frac{\Omega_{1} a_{1} \zeta}{p_{1}},
$$

and

$$
\Phi>\frac{\Omega_{2} a_{2} \zeta}{p_{2}}
$$

where these results are obtained through $\lambda_{1}^{(0)}<0$, and $\lambda_{2}^{(0)}<0$. The values of the normalised parameters are positive, then the eigenvalues $\lambda_{3}^{(0)}$, and $\lambda_{4}^{(0)}$ are negative. We consider $a_{1}=a_{2}=1, \Omega_{1}=0.0068, \Omega_{2}=0.012, p_{1}=$ $4.7 \times 10^{-8}, p_{2}=4.7 \times 10^{-5}$, and $\zeta=0.2($ table 1$)$. With these values we obtain that $E_{0}$ is linearly asymptotically stable for $\Phi>28936.17$. In other words, if $\Phi>28936.17$ the chemotherapeutic agent kills all cells, they will never recover. Stability of the non-cells state is however granted for a very large atypical value of the infusion rate $\Phi$.

We also consider the equilibrium $E_{1}(\bar{g}, 0, \bar{n}, \bar{Q})$, representing the complete elimination of glioma cells in the normalised model, but preserving glial and neuron cells. This equilibrium is obtained by the solution of

$$
\begin{aligned}
\Omega_{1} \bar{g}(1-\bar{g})-\frac{p_{1} \bar{g} \bar{Q}}{a_{1}+\bar{g}} & =0, \\
-\frac{p_{3} \bar{n} \bar{Q}}{a_{3}+\bar{n}} & =0, \\
\Phi-\zeta \bar{Q} & =0
\end{aligned}
$$

where we obtain $\bar{n}=0$ and $\bar{Q}=\Phi \zeta^{-1}$. Thus, the equilibrium $E_{1}(\bar{g}, 0, \bar{n}, \bar{Q})$ is given by $E_{1}\left(\bar{g}, 0,0, \Phi \zeta^{-1}\right)$, meaning that all neurons are also eliminated.

The first equation of (15) can be rewritten as

$$
\bar{g}^{2}+\left(a_{1}-1\right) \bar{g}-a_{1}+\frac{p_{1} \Phi}{\Omega_{1} \zeta}=0,
$$

with solution

$$
\begin{aligned}
\bar{g}= & \frac{1}{2}\left\{1-a_{1} \pm\left[\left(a_{1}-1\right)^{2}+4\left(a_{1}\right.\right.\right. \\
& \left.\left.\left.-p_{1} \Phi / \Omega_{1} \zeta\right)\right]^{1 / 2}\right\} .
\end{aligned}
$$

In this way, we verify that $\bar{g}$ has a null solution when $p_{1} \Phi / \Omega_{1} \zeta=a_{1}$, and a real, positive and not null solution when $p_{1} \Phi / \Omega_{1} \zeta<a_{1}$. Using the parameters of Table 2, $\bar{g}$ has a real, positive and non null solution when $\Phi<$ 28936.17.

Calculating a lower band for the value of $\Phi$ for which the equilibrium $E_{1}\left(\bar{g}, 0,0, \Phi \zeta^{-1}\right)$ is stable, we determine the stability of this equilibrium. The eigenvalues of the Jacobian matrix are

$$
\begin{aligned}
\lambda_{1}^{(1)} & =\Omega_{1}(1-2 \bar{g})-\frac{p_{1} a_{1} \Phi}{\zeta\left(a_{1}+\bar{g}\right)^{2}}, \\
\lambda_{2}^{(1)} & =\Omega_{2}-\beta_{2} \bar{g}-\frac{p_{2} \Phi}{\zeta a_{2}}, \\
\lambda_{3}^{(1)} & =-\frac{p_{3} \Phi}{\zeta a_{3}} \\
\lambda_{4}^{(1)} & =-\zeta .
\end{aligned}
$$

In order to ensure the stability of $E_{1}\left(\bar{g}, 0,0, \Phi \zeta^{-1}\right)$ it is necessary

$$
p_{1} \Phi \zeta^{-1}>\frac{\Omega_{1}(1-2 \bar{g})\left(a_{1}-\bar{g}\right)^{2}}{a_{1}},
$$

and

$$
p_{2} \Phi \zeta^{-1}>a_{2}\left(\Omega_{2}-\beta_{2} \bar{g}\right)
$$

where these results are obtained through $\lambda_{1}^{(1)}<0$, and $\lambda_{2}^{(1)}<0$. The values of the dimensionless parameters are positives, then the eigenvalues $\lambda_{3}^{(1)}$, and $\lambda_{4}^{(1)}$ are negatives.

For $a_{1}=1.0$ (Table 2) Eq. (22) is satisfied for all $\bar{g} \geq$ 0.5. Considering $a_{2}=1.0, \Omega_{2}=0.012, p_{2}=4.7 \times 10^{-5}$, $\zeta=0.2$, and $\beta_{2}=1.8 \times 10^{-3}$ (Table 2) in Eq. (23) we have $\Phi>51.064-7.660 \bar{g}$. As a result, for $E_{1}\left(\bar{g}, 0,0, \Phi \zeta^{-1}\right)$ the system presents an asymptotically stable equilibrium for $\Phi>43.189$. Therefore, for realistic values of the infusion rate $43.189<\Phi<28979.255$, we should expect that glioma can be eliminated, i. e., $c \leq 10^{-11}$. Doing similar analyses in the non-normalised system, we observe that the equilibrium $E(\bar{g}, 0, \bar{n}, \bar{Q})$ is also stable for $43.189<\Phi<28979.255$.

We construct the parameter space shown in Fig. 4 to obtain a picture of the stability according to parameters related with the chemotherapy. We can observe three regions. Region I represent parameter which the glioma cells kill the glial cells and neurons, region II represents parameters for which the equilibrium $E_{1}\left(\bar{g}, 0,0, \Phi \zeta^{-1}\right)$ is locally stable, and in region III represents parameters for which the equilibrium $E_{0}\left(0,0,0, \Phi \zeta^{-1}\right)$ is locally stable. The region II shows that glioma can be eliminated without the elimination of the glial cells. However, the longer the duration of treatment, the larger the decrease in the neural population. It is therefore vital to understand what are the optimal parameters for which $c \leq 10^{-11}$ is achieved in the shortest time.

A strongly desired equilibrium is $E_{2}(\bar{g}, 0, \bar{n}, 0)$. In this case we have $\bar{g}=1$ and $\bar{n}$ has a constant value. The 


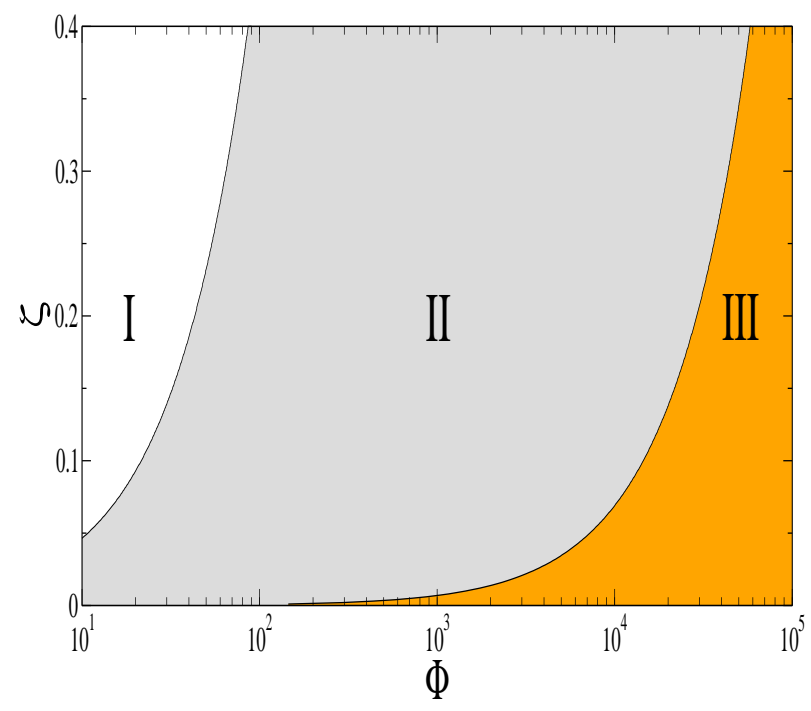

Figure 4: (Colour online) Parameter space $\zeta$ versus $\Phi$ : in the region I the glioma cells kill the glial cells and neurons, the region II shows which the equilibrium $E_{1}\left(\bar{g}, 0,0, \Phi \zeta^{-1}\right)$ is locally stable, and in the region III the equilibrium $E_{0}\left(0,0,0, \Phi \zeta^{-1}\right)$ is locally stable.

eigenvalues are $\lambda_{1}=-\Omega_{1}, \lambda_{2}=\Omega_{2}-\beta_{2}, \lambda_{3}=0$, and $\lambda_{4}=-\zeta$. Using the parameters given in Table 2 we obtain negative values for $\lambda_{1}$ and $\lambda_{4}, \lambda_{2}$ has a positive value, and $\lambda_{3}$ presents a null value. This equilibrium is an unstable saddle point. Then, if treatment is ceased without the complete elimination of the glioma cells $(c=0)$, the glioma concentration in our model increases.

\section{Glioma elimination}

Here, we study the performance of our model to understand what are the conditions such that glioma concentration in the normalised model reaches levels related to no glioma $\left(c \leq 10^{-11}\right)$, while glial and neuron cells concentration are kept high.

Figure 3 shows a case for eliminated glioma. However, the neurons concentration is decreased by $1.5 \%$ when the tumour has significantly decreased. In this case, glioma is eliminated, but a significant population of neurons are damaged. For this reason we optimise the values of the chemotherapeutic agents in order not only to minimise the impact on neurons but also to maximise the effect of the drug on glioma cells. Our aim is to understand how the neuron population is when $c \leq 10^{-11}$. The therapeutic implication for neurons is shown in Figure 5, the neuron concentration (colour bar) when $c \leq 10^{-11}$, as a function of the parameters $\alpha$ and $\Phi$. In this case, we consider a chemotherapy delivered continuously. The region of $\alpha$ and $\Phi$ values responsible for the reduction of approximately $2 \%$ in the neuron concentration (yellow online) is $0.96 \leq n \leq$ 0.98 , while the region $0.80 \leq n \leq 0.84$ (dark blue online) presents approximately a reduction of $17 \%$. This region of parameters causes a large rate of death of the glias (large $\dot{g})$. The chemotherapy rate $\Phi$ also contributes to this low level of $n$.

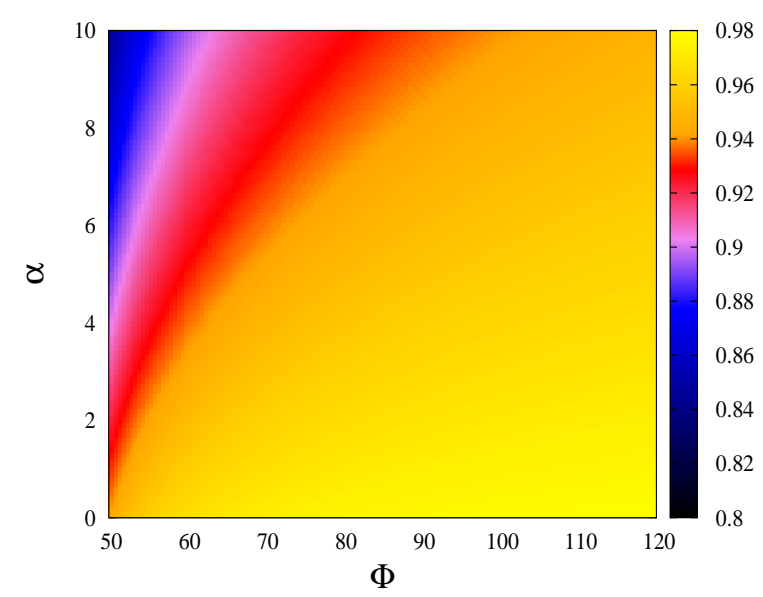

Figure 5: (Colour online) Neuron concentration as a function of $\alpha$ versus $\Phi$, where $g(0)=0.99, c(0)=0.01, n(0)=0.99$, and $Q(0)=$ 0.0 . The colour bar represents the value of the neuron concentration, $n$, after a successful chemotherapy.

Since that glial cells provide support functions for the neurons, we also analyse the concentration of the glial cells with the chemotherapy treatment. For the parameter values showed in Fig. 5, the percentage of glial cells remains larger than 95\%. Equations (7) show that the glial cells equation does not depend on the parameter $\alpha$, but it depends on the parameter $\Phi$ due to $Q$. When $\Phi$ increases, we verify that $c$ decreases. However, there is not a significant variation in $g$ due to the lifetime glioma $\tau$ according to the chemotherapy agent rate. In other words, increasing the value of $\Phi$ the lifetime of glioma quickly decreases, and in this time interval the glial cells concentration does not have a significant alteration, due to the fact that the glial cells are able to recover to their initial state.

Figure 6 shows the time $\tau$ to achieve suppression of glioma as a function of $\Phi$. There is a power-law relation of the type $\tau \propto \Phi^{\sigma}$, with $\sigma=-12.36$ for $\Phi \leq 60$ $\mathrm{mg}\left(\mathrm{m}^{2} \cdot \text { day }\right)^{-1}$ and $\sigma=-1.41$ for $\Phi \geq 60 \mathrm{mg}\left(\mathrm{m}^{2} \cdot \text { day }\right)^{-1}$. This power-law shows that a significant decrease in $\tau$ happens if $\Phi \leq 60 \mathrm{mg}\left(\mathrm{m}^{2} \cdot \text { day }\right)^{-1}$, whereas little modification in $\tau$ happens if $\Phi>60 \mathrm{mg}\left(\mathrm{m}^{2} \text {.day }\right)^{-1}$. Therefore, the optimal way of reducing the time of treatment by using the minimal amount of $\Phi$ is obtained if $\Phi \approx 60 \mathrm{mg}\left(\mathrm{m}^{2} \cdot \text { day }\right)^{-1}$. Looking at Fig. 5, neurons will also be significantly preserved if $\alpha \leq 2$. The same scalings are obtained if another $\alpha$ if considered. The chemotherapeutic agent which provides the quickest is $\Phi \leq 60 \mathrm{mg}\left(\mathrm{m}^{2} \cdot \text { day }\right)^{-1}$. Treatment will cause less impact on neural population if the individual being treated (characterises by a particular $\alpha$ ) is provided with an infusion rate $\Phi$ such that the point $(\Phi, \alpha)$ falls in the yellow region in Fig. 5 .

Often chemotherapy treatments are delivered in cycles, where drugs are repeatedly applied for a short time. In the case of glioma and temozolomide, after radiation therapy, 


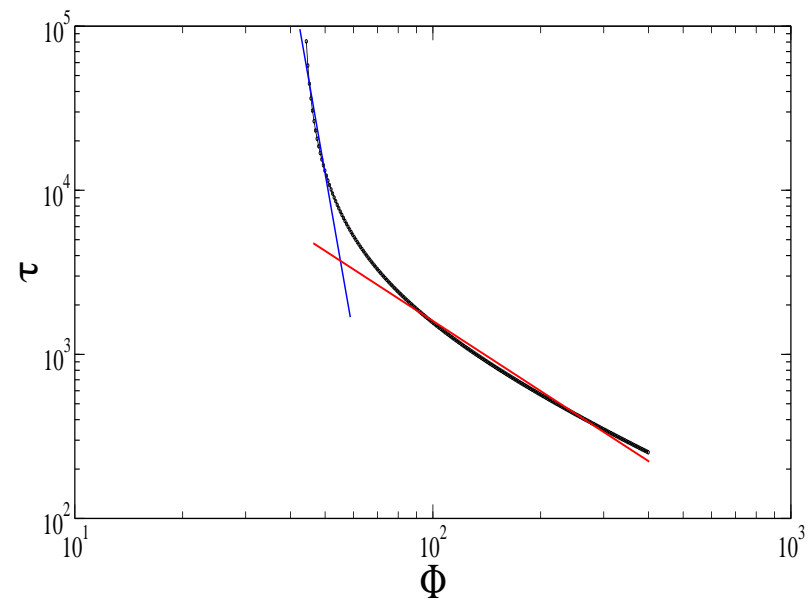

Figure 6: $\tau$ versus $\Phi$, and the same values as Figure 5 . The slope for $\Phi \leq 60 \mathrm{mg}\left(\mathrm{m}^{2} \cdot \text { day }\right)^{-1}$ is about -12.36 , and for $\Phi \geq 60$ $\mathrm{mg}\left(\mathrm{m}^{2} \cdot \text { day }\right)^{-1}$ the slope is about -1.41 .

the drug is delivered 5 days on, and 23 days off. There have been clinical experiences with temozolomide considering pulsed chemotherapy in patients with glioma (Friedman et al., 2000; Pace et al., 2003). The patients received radiotherapy before the chemotherapy. It was verified that temozolomide chemotherapy was a valid option.

Figure 7(a) shows the drug injection pattern for pulsed chemotherapy. We consider 5 days on with $\Phi=400$ $\mathrm{mg}\left(\mathrm{m}^{2} \cdot \text { day }\right)^{-1}$, and 23 days off. Figures (b), (c), and (d) exhibit the temporal evolution of the concentration of glial cells, glioma cells, and neurons, respectively. There is not a relevant decrease in the concentration of glial cells (Fig. $7 \mathrm{~b}$ ), but the concentration of glioma cells are going to a suppressed state (Fig. 7c). Whereas, the concentration of neuron decreases around only slightly.
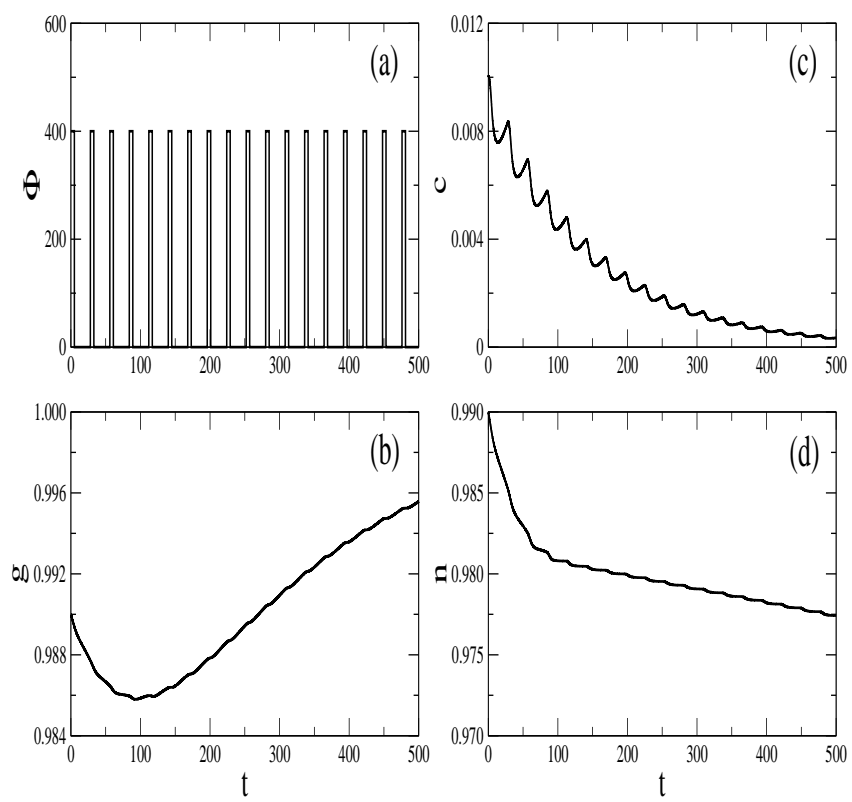

Figure 7: Temporal evolution of the (a) chemotherapy infusion, concentration of (b) glial cells, (c) glioma cells, and (d) neurons.

\section{Conclusions}

We proposed a mathematical model for the evolution of a brain tumour under the attack of chemotherapeutic agents. Our model describes the interactions among glial cells, neurons, and glioma, with a chemotherapy to suppress the brain tumour. The novelty in this model is the glial effect on the neurons.

We studied some aspects of the dynamics of glioma growth, as well as, we analysed its suppression and elimination varying parameters of the system. The main target of treatment is the decrease in the number of glioma cells. A successful chemotherapy eliminates all the glioma cells minimising the neurons and glial cells injury. Through local stability we found a range of values for the infusion rate $(43.189<\Phi<28979.255)$ that allows for the elimination of glioma, as well as, the glioma will not return. As a matter of fact the temozolomide is a chemotherapeutic drug used for brain tumour, where the infusion rate is $75<\Phi<200\left(\mathrm{mg}\left(\mathrm{m}^{2} \text {. day }\right)^{-1}\right)$ (Wick et al., 2009). According to our model the rate would kill all the glioma cells, and in addition it would preserve high levels of neural population. The range of the values for the infusion rate is clinically relevant because it reveals the effectiveness of the treatment strategies by administration of chemotherapeutic drugs. Brock and collaborators (Brock et al., 1998) used an extended continuous oral schedule of temozolomide against gliomas. They verified which patients with recurrent glioma were the main group in that tumour responses were seen. Clinical studies conducted by the Cancer Research Campaign (London, United Kingdom) demonstrated which temozolomide has important efficacy an acceptable safety profile in the treatment of patients with glioma (Friedman et al., 2000). New strategies have been developed aiming health benefits of patients, that is the elimination of glioma cells (Minniti et al., 2009). Regardless whether doctors will use the optimal rates obtained from our model, our work can help doctors to access the risks of a treatment on an individual basis. For example, Figure 5 shows that depending on the value of $\alpha$ (a parameter that depends on the individual) the range of vales for the infusion rate can be larger as smaller for an optimal application.

We realised numerical simulations and obtained values of the infusion of chemotherapeutic agents in that the glioma growth is eliminated within the shortest time. We considered a continuous infusion throughout the entire period of simulation. However, $\Phi$ can be time dependent to simulate pulsed chemotherapy.

Our main result was to show that chemotherapy can be applied mitigating the side effects of drugs on the neurons death, if the appropriated rate is used. Mechanisms of chemotherapy-induced neurotoxicicty have not been completely elucidated. Wick and collaborators (Wick et al., 2004) analysed the effects of four chemotherapeutic drugs (lomustine, cisplatin, topotecan, and vincristine) on neurons and glias derived from rats. They observed neuron 
death in a concentration-dependent manner. Gong and collaborators (Gong et al., 2011) also analysed the neurotoxicity due to chemotherapy against glioma. They concluded that newer chemotherapy agents (proteasome inhibitor bortezomib, and epidermal growth factor receptor tyrosine kinase inhibitor erlotinib) are effective against glioma cells, producing minimal effects on neurons. Older drugs (temozolomide, and cisplatin) are more toxic for neurons than for glioma cells. With this in mind, it is possible to obtain an appropriate infusion rate if we have the time evolution of the neuron concentration when a chemotherapy agent is applied in the brain.

Our model suggests that the death of one glioma may occur after 20 weeks. On the other hand, by 10 weeks the model presents a decrease about $70 \%$ in the concentration of glioma cells. For instance, the temozolomide has good penetration into brain, and Oshiro and collaborators reported the clinical efficacy of temozolomide in patients with glioma (Oshiro et al., 2009). They observed the reduction of $\geq 50 \%$ in tumour size for $\geq 4$ weeks of the $40 \%$ of patients. The effect depends on the infusion rate that is associated with the drugs and methods of treatments. Moreover, the dose rate also depends on the side effects of chemotherapy on the body, due to the fact that different drugs cause different effects (Brock et al., 1998).

We believe that our model, which consider the interaction between neuron and glia, constitutes an important step toward developing strategies for glioma treatment. The understanding of tumour growth dynamics may help in the treatment of diseases.

In future works, we plan to study this biological process considering nonlinear time and space models to describe the spatiotemporal evolution patterns of glioma.

\section{Acknowledgments}

This study was possible by partial financial support from the following Brazilian government agencies: Fundação Araucária, EPSRC-EP/I032606/1, CNPq, CAPES and Science Without Borders Program - Process n ${ }^{\circ} 17656125$, $\mathrm{n}^{\circ}$ 99999.010583/2013-00 and $\mathrm{n}^{\circ} 245377 / 2012-3$.

\section{References}

Adam, J.A., Bellomo, N., 1996. A Survey of Models for TumorImmune System Dynamics. Birkhäuser Basel, Boston.

Alarcón, T., Byrne, H.M., Maini, P.K., 2003. A cellular automaton model for tumour growth in inhomogeneous environment. J. Theor. Biol. 225,v257-274.

Alberts, B., Bray, D., Lewis, J., Raff, M., Roberts, K., Watson, J.D., 1994. Molecular Biology of The Cell. Garland Publishing, New York.

Allen, N.J., Barres, B.A., 2009. Glia - more than just brain glue. Nature 457, 5, 675-677.

Aroesty, J., Lincoln, T., Shapiro, N., Boccia, G., 1973. Tumor growth and chemotherapy: mathematical methods, computer simulations, and experimental foundations. Math. Biosci. 17, 243-300.

Azevedo, F.A.C., Carvalho, L.R.B., Grinberg, L.T., Farfel, J.M., Ferretti, R.E.L., Leite, R.E.P., Filhos, W.J., Lent, R., HerculanoHouzel, S., 2009. Equal numbers of neuronal and nonneuronal cells make the human brain an isometrically scaled-up primate brain. J. Comp. Neur. 513, 532-541.

Borges, F.S., Iarosz, K.C., Ren, H.P., Batista, A.M., Baptista, M.S., Viana, R.L., Lopes, S.R., Grebogi, C., 2014. Model for tumour growth with treatment by continuous and pulsed chemotherapy. BioSystems 116, 43-48.

Brock, C.S., Newlands, E.S., Wedge, S.R., Bower, M., Evans, H., Colquhoun, I., Roddie, M., Glaser, M., Bramptons, M.H., Rustin, G.J.S., 1998. Phase I trial of temozolomide using an extended continuous oral schedule. Cancer Res. 58, 4363-4367.

Bulstrode, H., Jones, L.M., Siney, E.J., Sampson, J.M., Ludwig, A., Gray, W.P., Willaime-Morawek, S., 2012. A-disintegrin and metalloprotease (ADAM) 10 and 17 promote self-renewal of brain tumor sphere forming cells. Canc. Lett. 326, 79-87.

Burrel, R.A., McGranahan, N., Bartek, J., Swanton, C., 2013. The causes and consequences of genetic heterogeneity in cancer evolution. Nature 501, 338-345.

Cattani, C., Ciancio A., 2008. Qualitative analysis of second-order models of tumor-immune system competition. Math. Comput. Model. 47, 1339-1355.

Clarke, G., Collins, R.A., Leavitt, B.R., Andrews, D.F., Hayden, M.R., Lumsden, C.J., Mclnnes, R.R., 2000. A one-hit model of cell death in enherited neuronal degenerations. Nature 406, 195199.

Cuddapah, V.A., Robel, S., Watkins, S., Sontheimer, H., 2014. A neurocentric perspective on glioma invasion. Nature Rev. Neurosci. $15,455-465$.

Fieldes, R.D., 2006. Advances in understanding neuron-glia interactions. Neur. Glia Biol. 2, 23-26.

Friedman, H.S., Kerby, T., Calvert, H., 2000. Temozolomide and treatment of malignant glioma. Clin. Cancer Res. 6, 2585-2597.

Gao, X., Li, C., 2014. Nanoprobes visualizing gliomas by crossing the blood brain tumor barrier. Small 10, 3, 426-440.

Gerlee, P., Nelander, S., 2012. The impact of phenotypic switching on glioblastoma growth and invasion. PLOS ONE Comp. Bio. 8, 6, e1002556.

Gong, X., Schwartz, P.H., Linskey, M.E., Bota, D.A., 2011. Neural stem/progenitors and glioma stem-like cells have differential sensitivity to chemotherapy. Neurology 76, 1126-1134.

Ghosh, A., Chaudhuri, S., 2010. Microglial action in glioma: A boon turns bane. Immunol. Lett. 131, 3-9.

Glees, P., 1955. Neuroglia: morphology and function. Blackwell, Oxford.

Goodenberger, M. L., Jenkins, R. B., 2012. Genetics of adult glioma. Cancer Genetics 205, 613-621.

Hahn, W.C., Weinberg, R.A., 2002. Modelling the molecular circuitry of cancer. Nat. Rev. Canc. 2, 331-341.

Harpold, L.P.H., Alvord Jr, E.C., Swanson, K.R., 2007. The evolution of mathematical modelling of glioma proliferation and invasion. J. Neuropathol. Exp. Neurol. 66, 1, 1-9.

Hirt, C., Papadimitropoulos, A., Mele, V., Muraro, M. G., Mengus, C., Iezzi, G., Terracciano, L., Martin, I., Spagnoli, G. C., 2014. "In vivo" 3D models of tumor-immune system interaction. Adv. Drug Deliv. Reev. (in press).

Holling, C.S., 1965. The functional response of predator to prey density and its role in mimicry and population regulation. Mem. Ent. Sec. Can. 45, 1-60.

Inaba, N., Kimura, M., Fujioka, K., Ikeda, K., Somura, H., Akiyoshi, K., Inoue, Y., Nomura, M., Saito, Y., Saito, H., Manome, Y., 2011. The effect of PTEN on proliferation and drug-, and radiosensitivity in malignant glioma cells. Anticancer Res. 31, 1653-1658.

Kronik, N., Kogan, Y., Vainstein, V., Agura, Z., 2007. Improving alloreactive CTL immunotherapy for malignant gliomas using a simulation model of their interactive dynamics. Cancer Immunol. Immunother. DOI 10.1007/s00262-007-0387-z.

Louis, D.N., Ohgaki, H., Wiestler, O.D., Cavenee, W.K., Burger, P.C., Jouvet, A., Scheithauer, B.W., Kleihues, P., 2007. The 2007 WHO classification of tumours of the central nervous system. Acta Neuropathol. 114, 97-109.

Menchón, S.A., Condat, C.A., 2008. Cancer growth: predictions of a realistic model. Phys. Rev. E 78, 022901. 
Meyer-Franke, A., Kaplan, M.R., Pfrieger, F.W., Barres, B.A., 1995. Characterization of the signaling interactions that promote the survival and growth of developing retinal ganglion cells in culture. Neuron 15, 805-819.

Minniti, G., Muni, R., Lanzetta, G., Marchetti, P., Enrici, R.M., 2009. Chemotherapy for glioblastoma: current treatment and future perspectives for cytotoxic and targeted agents. Anticancer Res. 29, 5171-5184.

Nakae, K., Ikegaya, Y., Ishikawa, T., Oba, S., Urakubo, H., Koyama M., Ishii, S., 2014. A statistical method of identifying interactions in neuron-glia systems based on functional multicell $\mathrm{Ca} 2+$ imaging. Plos Comput. Biol. 10, e1003949.

Oshiro, S., Tsugu, H., Komatsu, F., Ohmura, T., Ohta, M., Sakamoto, S., Fukushima, T., Inoue, T., 2009. Efficacy of temozolomide treatment in patients with high-grade glioma. Anticancer Res. 29, 911-918.

Otis, T.S., Sofronie, M.V., 2008. Glia get excited. Nat. Neurosci. 11, 379-380.

Pace, A., Vidiri, A., Galiè, Carosi, M., Telera, S., Cianciulli, A.M., Canalini, P., Giannarelli, D., Jandolo, B., Carapella, C.M., 2003. Ann. Oncol. 14, 1722-1726.

Pei, Y., Chen, L., Zhang, Q., Li, C., 2005. Extinction and permanence of one-prey multi-predators of Holling type II function response system with impulsive biological control. J. Theor. Biol. 235, 4, 495-503.

Pinho, S.T.R., Freedman, H.I., Nani, F.A., 2002. A chemotherapy model for the treatment of cancer with metastasis. Math. Comput. Model. 36, 773-803.

Pinho, S.T.R., Barcelar, F.S., Andrade, R.F.S., Freedman, H.I., 2013. A mathematical model for the effect of anti-angiogenic therapy in the treatment of cancer tumours by chemotherapy. Nonlinear Analys.: Real World Applicat. 14, 815-828.

Ribba, B., Kaloshi, G., Peyre, M., Ricard, D., Calvez, V., Tod, M. Cajavec-Bernard, B., Idbaih, A., Psimaras, D., Dainese, L., Pallud, J., Cartalat-Carel S., Delattre, J.-Y., Honnorato, J., Grenier, E., Ducray, F., 2012. A tumor growth inhibition model for lowgrade glioma treated with chemotherapy or radiotherapy. Clin. Cancer Res. 18, 5071-5080.

Rzeski, W., Pruskil, S., Macke, A., Felderhoff-Mueser, U., Reiher, A.K., Hoerster, F., Jansma, C., Jorosz, B., Stefovska, V., Bittigau, P., Ikonomidou, C., 2004. Anticancer agents are potent neurotoxins in vitro and in vivo. Ann. Neurol. 56, 351-360.

Said, R., Abdel-Rehim, M., Sadeghi, B., Al-Hashemi, S., Hassan, Z., Hassan, M., 2007. Cyclophosphamide pharmacokinetics in mice: a comparison between retro orbital sampling versus serial tail vein bleeding. The Open Pharmacol. J. 1, 30-35.

Scaleranpdi, M., Romano, A., Pescarmona, G.P., Delsanto, P.P., Condar, C.A., 1999. Nutrient competition as a determinant for cancer growth. Phys. Rev. E 59, 2206-2217.

Schuette, W., 2004. Treatment of brain metastases from lung cancer: chemotherapy. Lung. Canc. 45, 253-257.

Shaham, S., 2005. Glia-neuron interactions in nervous system function and development. Curr. Top. Dev. Biol. 69, 39-66.

Spratt, J.S., Spratt, T.L., 1964. Rates of growth of pulmonary metastases and host survival. Ann. Surg. 159, 161-171.

Srimanee, A., Regberg, J., Hallbrink, M., Kurrikoff, K., Veiman, K.-L., Vajragupta, O., Langel, Ü., 2014. Peptide-base delivery of oligonucleotides across blood-brain barrier model. Int. J. Pept. Res. Ther. 20, 169-178.

Stupp, R., Mason, W.P., Van den Bent, M.J., Weller, M., Fisher, B., Taphoorn, M.J.B., Belanger, K., Brandes, A.A., Marosi, C., Bogdahn, U., Curschmann, J., Janzer, R.C., Ludwin, S.K., Gorlia, T., Allgeier, A., Lacombe, D., Cairncross, J.G., Eisenhauer, E., Mirimanoff, R.O., 2005. Radiotherapy plus concomitant and adjuvant temozolomide for glioblastoma. N. Engl. J. Med. 352, 987-996.

Tiwari, V., Guan, Y., Raja, S.N., 2014. Modulating the delicate glianeuronal interactions in neuropathic pain: Promises and potential caveats. Neurosci. Biobehav. R. 45, 19-27.

Weille, J., 2014. On the genesis of neuroblastoma and glioma. Int. J. Brain Sci. 217503, 1-14.
Wheldon, T.E.. 1988. Mathematical Models in Cancer Research. Taylor and Francis, London.

Wick, A., Wick, W., Hirrlinger, J., Gerhardt, E., Drigen, R., Dichgans, J., Weller, M., Schulz, J.B., 2004. Chemotherapy-induced cell death in primary cerebellar granule neurons but not in astrocytes: in vitro paradigm of differential neurotoxicity. J. Neurochem. 91, 1067-1074

Wick, W., Platten, M., Weller, M., 2009. New (alternative) temozolomide regimens for the treatment of glioma. Neuro. Oncol. 11(1), 69-79.

Wolpert, L., Beddington, R., Jessel, T., Lawrence, P., Meyerowitz, E., Smith, J., 2002. Principles of desenvolviment. Oxford University Press.

Yang, F-Y., Lin, Y-L., Chou, F.-I., Lin, Y.-C., Liu, Y.-W.H., Chang, L.-W., Hsieh, Y.-L., 2014. Pharmacokinetics of BPA in gliomas with ultrasound induced blood-brain barrier disruption as measured by microdialysis. PLOS ONE 9, 6, e100104.

Yang, I., Hand, S.J., Kaur, G., Crane, C., Parsa, A.T., 2010. The role of microglia in central nervous system immunity and glioma immunology. J. Clin. Neurosci. 17, 6-10.

Ye, Z.-C., Sontheimer, H., 1999. Glioma cells release excitotoxic concentrations of glutamate. Cancer Res. 59, 4383-4391. 